\title{
Transplant psychiatry
}

\author{
SG Potts \\ Consultant Psychiatrist, Department of Psychological Medicine, Royal Infirmary of Edinburgh, Edinburgh, UK
}

\begin{abstract}
Transplant units increasingly recognise a need for assistance from psychiatrists and psychologists in the assessment and management of potential transplant recipients and live donors. This arises from the various known associations between mental disorder and the need for transplantation; the intensifying requirement to select carefully among the potential recipients and donors of scarce human organs; and the drive to maximise transplant outcomes by optimising all aspects of treatment after surgery. There is good evidence that careful, protocol-guided selection among potential candidates for transplantation with alcoholic liver disease helps ensure outcomes at least as good as for other forms of liver disease. The evidence base in other areas is less robust, but the principles guiding the psychiatric assessment are broadly the same. There is an increasing need for psychiatric assessment of potential live organ donors, in order to minimise the risks they run, and in the case of altruistic donation this is now mandatory in UK law.
\end{abstract}

Published online September 2009

Correspondence to SG Potts, Department of Psychological Medicine, Royal Infirmary of Edinburgh, Edinburgh EHI6 4SA, UK

tel. +44 (0) I 3 I 242 I398

e-mail

stephen.potts@luht.scot.nhs.uk

KEYWORDS Donor, mental disorder, psychiatry, recipient, selection, transplant

DECLARATION OF INTERESTS No conflict of interests declared.

\section{ASSOCIATIONS BETWEEN THE NEED FOR TRANSPLANT, MENTAL DISORDER AND OTHER BEHAVIOURS}

\section{Mental disorder}

Mental disorder is associated with the need for solid organ transplant in a number of ways (see Table I). In the UK, liver transplant is most commonly undertaken for diseases arising directly from the deliberate intake of hepatotoxins, whether chronically, as in alcoholic liver disease (ALD), or acutely, as in paracetamol poisoning. Alcohol dependence and the harmful use of alcohol are recognised mental disorders, and paracetamol overdoses are a common manifestation of mental disorder. Less commonly, heart transplants may be undertaken for alcoholic cardiomyopathy. The second most common chronic condition requiring liver transplants is viral hepatitis, which is commonly an indirect consequence of another behaviour that may amount to mental disorder, namely intravenous drug misuse. End-stage renal failure (ESRF), although responsible for by far the most procedures among solid organ transplants, is less commonly a consequence of mental disorder. It can arise, however, as a result of psychiatric treatment, in a small proportion of patients on long-term lithium for bipolar disorder.

\section{Other behaviours}

There are other aspects of behaviour that may generate a need for transplant and, although not mental disorders in themselves, may be associated with mental disorder and are open to psychological treatment. They may therefore benefit from an assessment by a psychologist or psychiatrist as part of a transplant work-up. These can broadly be
TABLE I Mental disorder generating a need for transplants

\begin{tabular}{|c|c|c|}
\hline Organ & Mental disorder & Pathology \\
\hline \multicolumn{3}{|l|}{ Directly } \\
\hline $\begin{array}{l}\text { Liver } \\
\text { - chronic } \\
\text { - acute }\end{array}$ & $\begin{array}{l}\text { Alcohol dependence/ } \\
\text { misuse } \\
\text { Paracetamol poisoning }\end{array}$ & $\begin{array}{l}\text { Alcoholic liver disease } \\
\text { Fulminant hepatic failure }\end{array}$ \\
\hline Heart & $\begin{array}{l}\text { Alcohol dependence/ } \\
\text { misuse }\end{array}$ & $\begin{array}{l}\text { Alcoholic } \\
\text { cardiomyopathy }\end{array}$ \\
\hline \multicolumn{3}{|l|}{ Indirectly } \\
\hline $\begin{array}{l}\text { Liver } \\
\text { - chronic }\end{array}$ & Intravenous drug misuse & Hepatitis $B / C$ infection \\
\hline \multicolumn{3}{|c|}{ As a consequence of psychiatric treatment } \\
\hline Kidney & $\begin{array}{l}\text { Long-term lithium for } \\
\text { mood disorder }\end{array}$ & $\begin{array}{l}\text { End-stage renal failure } \\
\text { due to lithium }\end{array}$ \\
\hline
\end{tabular}

divided into maladaptive non-compliance with medical treatments and behavioural risk factors such as smoking, poor diet, obesity and lack of exercise (see Table 2).

Non-compliance with treatments may be a manifestation of a mental disorder such as depression or anxiety (for example in the case of a patient so fearful of hypoglycaemia that he runs his blood sugar too high). In these circumstances effective treatment of the mental disorder may improve compliance and thus medical outcome.

\section{PREDICTING PROGNOSIS}

Some mental disorders, such as intravenous drug misuse, have intrinsically poor prognoses, in terms of mortality and major mental and physical morbidity, even without 
TABLE 2 Other behaviours generating a need for transplants

\begin{tabular}{|c|c|c|}
\hline Organ & Behaviour & Pathology \\
\hline \multicolumn{3}{|c|}{ Non-compliance } \\
\hline Kidney & $\begin{array}{l}\text { Non-compliance } \\
\text { with treatment } \\
\text { - for diabetes } \\
\text { - for hypertension }\end{array}$ & $\begin{array}{l}\text { Diabetic nephropathy } \\
\text { Hypertensive nephropathy }\end{array}$ \\
\hline Heart & $\begin{array}{l}\text { Non-compliance } \\
\text { with treatment } \\
\text { - for hypertension }\end{array}$ & Hypertensive heart disease \\
\hline \multicolumn{3}{|c|}{ Behavioural risk factors } \\
\hline Lung & Smoking & $\begin{array}{l}\text { Chronic obstructive } \\
\text { pulmonary disease } \\
\text { (COPD) }\end{array}$ \\
\hline Heart & $\begin{array}{l}\text { Smoking, diet, } \\
\text { obesity, exercise }\end{array}$ & Coronary artery disease \\
\hline Liver & Diet, obesity & $\begin{array}{l}\text { Non-alcoholic fatty liver } \\
\text { disease }\end{array}$ \\
\hline
\end{tabular}

TABLE 3 Mental disorder, behavioural abnormalities and transplant: prognostic questions

\begin{tabular}{|l|}
\hline $\begin{array}{l}\text { For recurrent mental disorder currently } \\
\text { in remission (e.g. severe depression): }\end{array}$ \\
\hline Risk of relapse \\
\hline $\begin{array}{l}\text { Is the risk of relapse increased by transplantation } \\
\text { (stress, steroids, etc)? }\end{array}$ \\
\hline Will a relapse endanger survival of the patient or the graft? \\
\hline Effect of mental disorder upon transplant candidate's: \\
\hline Understanding \\
\hline Resilience \\
\hline Compliance with post-transplant regime \\
\hline Risk of self-harm and suicide \\
\hline
\end{tabular}

the added impact of transplantation. For this reason they are usually considered absolute contraindications to transplantation, in a manner exactly analogous to physical conditions with equivalently poor prognoses. More commonly, judgements are required about less clear-cut aspects of prognosis in transplant candidates with co-morbid mental disorders. These are outlined in Table 3.

The process of transplantation is very demanding of patients and those around them. It requires a period of intense and often invasive investigation, short-notice hospitalisation, major surgery, immunosuppression and life-long follow-up. Outcomes are best when transplant candidates understand all these requirements, have the personal resilience (and social support) necessary to cope with them, and the ability and willingness to comply with the limitations on and requirements of them after transplant - such as keeping appointments, taking medication, observing dietary restrictions and abstaining from cigarettes, alcohol and drugs.
Mental disorders have the potential to limit understanding, resilience and compliance in a wide variety of ways. For example, a learning disability may impair understanding, chronic depression may limit resilience and substance misuse or personality disorder may impair compliance. In addition, many mental disorders are associated with radically increased rates of suicide and self-harm: this is, of course, most relevant when the need for transplant arises from suicidal or selfharming behaviour.

However, a dearth of data means that in most cases a judgement about prognosis is exactly that: a judgement, based on clinical experience and available evidence of prognosis in mental disorders not co-morbid with transplantation or the need for it.

\section{PSYCHIATRIC ASSESSMENTS IN TRANSPLANTATION}

\section{Selecting among recipients}

Much of the foregoing is also true of other highly technological medical and surgical treatments where there is no comparable demand for psychiatric assessment. This specific demand within transplant services arises for two reasons, both relating to the nature of the primary resource in all transplantation, which is, of course, the rate-limiting supply of human organs, rather than a potentially expandable funding stream. Although rates of both cadaveric and living donor transplant are rising in the UK as in most other countries, expanding demand outstrips this supply by a widening margin. The result is an ever-increasing number of patients who live (and die) on transplant waiting lists. Every transplant undertaken is thus both a potential benefit to the recipient, and an opportunity cost for all those left on the list. It therefore behoves transplant teams to ensure the best possible outcome for all transplants undertaken, and this is best achieved by only adding patients to transplant lists after a comprehensive assessment gives confidence about outcomes in terms of both graft and patient survival. For a growing proportion of patients, this assessment will include review by a psychiatrist and/or psychologist.

Secondly, because the resources in question (i.e. human organs) are essentially gifts from members of the public and their families, the public may claim a greater say in their allocation, than in, for example, the provision of intensive care beds. Transplant policies in general, and individual decisions in high-profile cases, carry the risk of reducing public willingness to donate, if they run counter to public wishes, especially in the context of conditions such as ALD. The best way to prevent this is to demonstrate robust efforts to discriminate among patients on the basis of likely outcome, rather than to allow discrimination against entire groups on the basis of a view that an illness such as ALD is self-inflicted or 
TABLE 4 Acute liver failure after paracetamol overdose: psychiatric contraindications to liver transplants

\begin{tabular}{|l|}
\hline Scottish Liver Transplant Unit guidelines \\
\hline Absolute contraindications \\
\hline I. Previous deliberate self-harm. Five or more lifetime \\
episodes unless previous events occurred many years ago. \\
\hline $\begin{array}{l}\text { 2. Substance misuse. Current substance dependence; } \\
\text { alternatively active substance misuse in a severe chaotic } \\
\text { fashion (may include intravenous drug use). }\end{array}$ \\
\hline $\begin{array}{l}\text { 3. Severe mental disorders. Chronic, severe, poor prognosis } \\
\text { mental illnesses especially if refractory to appropriate } \\
\text { treatment. Mental disorders in which the patient will have } \\
\text { no capacity to express wishes or understand circumstances. }\end{array}$ \\
\hline $\begin{array}{l}\text { 4. Declining transplant. Refusal to accept transplantation } \\
\text { when capacity is present. Consistently expressed wish to } \\
\text { die in the absence of explanatory mental illness. }\end{array}$ \\
\hline $\begin{array}{l}\text { 5. Poor compliance. Repeated non-compliance with medical } \\
\text { or psychiatric care. }\end{array}$ \\
\hline Relative contraindications \\
\hline $\begin{array}{l}\text { I. Previous self-harm. 2-4 lifetime episodes, especially when } \\
\text { associated with substance misuse, no significant life stresses/ } \\
\text { events and high lethality. }\end{array}$ \\
\hline $\begin{array}{l}\text { 2. Substance misuse. Current or recent but falling short of } \\
\text { dependence. Chaotic or intravenous drug use. }\end{array}$ \\
\hline $\begin{array}{l}\text { 3. Other significant/serious pervasive mental disorders such } \\
\text { as personality disorder or recurrent mood illness. }\end{array}$ \\
\hline
\end{tabular}

otherwise less worthy of treatment. In this respect transplant psychiatrists act as guardians against stigma.

Outcome data for liver transplantation in ALD are among the most comprehensive in this area, and show clearly that in appropriately assessed and selected patients, survival is at least as good as for other forms of chronic liver disease, and relapse into drinking is infrequent.

Protocols have been developed to guide assessment of potential transplant candidates with ALD: they generally list absolute contraindications (such as alcoholic hepatitis) and relative contraindications (such as a history of alcohol consumption within the last six months). In the UK the relevant document has been published by UK Transplant, through its Liver Advisory Group (see Further Reading).

Other national or local protocols guide assessment and listing decisions in other areas, such as liver transplant for hepatitis arising from illicit drug misuse, or urgent liver transplant in paracetamol poisoning (see Table 4). The adoption of such protocols reflects recognition that decision-making in such potentially contentious areas, where lives are at stake, must be open and consistent. However, there are some inconsistencies between protocols: a patient with ALD who continues to drink will not get access to a liver transplant list, while another with advanced hepatitis $C$ who is still taking prescribed methadone may - and via the same transplant unit.
Abstinence from a substance on which the patient is dependent is expected when the substance is alcohol, but not when it is a prescribed opiate.

Major psychiatric co-disorders, co-morbid with a need for transplant, are not necessarily contraindications for surgery. Some patients with schizophrenia, for example, may experience residual psychotic symptoms that do not impair their capacity to make decisions regarding surgery or their ability to cope afterwards. Distinguishing between these cases and those where psychiatric disorder definitely does pose a contraindication requires careful assessment by a transplant psychiatrist.

\section{Assessing live donors}

With cadaveric donation clearly providing insufficient organs, the UK and other countries have seen considerable increases in the use of live donors, mainly for kidneys, for obvious reasons. In most cases the transplant is between donors and recipients who are genetically and/or emotionally related, although paired transplants are now being undertaken in the UK, and pooled transplants (involving more than two donorrecipient pairs) are imminent. In addition, the use of non-directed 'altruistic' organ donors has become established since first becoming permissible under UK law in 2006.

Expanding the donor pool in these ways brings additional requirements for psychiatric or psychological assessment. All live donor transplantation involves major surgery, with attendant risks, on healthy individuals who do not stand to benefit directly. Potential donors are therefore assessed rigorously to ensure that these risks, whether physical or mental, are minimised. If, for example, a potential donor has a history of recurrent depressive illness, psychiatric assessment is indicated; and it may well conclude the risk of a depressive relapse after surgery is too great to justify it. Although necessarily paternalistic, these judgements are not intrinsically different from those made by cardiologists on potential donors with a cardiac history.

Practice varies between transplant units. Some require psychiatric or psychological assessment of all potential live donors; others are selective, using criteria such as those set out in Table 5.

The Human Tissue Act 2004 made non-directed donation possible for the first time in Britain. It also mandated psychiatric assessment in such cases, to be undertaken before any invasive pre-transplant investigation. The purpose of psychiatric review is not to approve or disapprove a potential donor's motives; instead it seeks to exclude from donation and its risks those whose wish to donate arises from an identifiable mental disorder, such as substance misuse, mood disorder or personality disorder. 
TABLE 5 Triggers to psychiatric referral in transplant assessment

\begin{tabular}{|l|}
\hline Aims: \\
\hline $\begin{array}{l}\text { To identify patients who may need additional support } \\
\text { before or after transplantation and to engage them with } \\
\text { appropriate support. }\end{array}$ \\
\hline $\begin{array}{l}\text { To identify patients for whom transplantation may be } \\
\text { inappropriate on psychosocial grounds. }\end{array}$ \\
\hline Factors to consider: \\
\hline $\begin{array}{l}\text { Active current substance misuse (where current means } \\
\text { within the last six months): } \\
\text { - alcohol - either high levels of consumption without } \\
\quad \text { harm; harmful use; or dependence } \\
\text { - illicit drug misuse } \\
\quad \text { dependence on prescribed medication (particularly, for } \\
\text { Evidence of problematic non-compliance with current } \\
\text { or previous medical treatments (including, for example, } \\
\text { insulin and other treatments for diabetes, antihypertensives, } \\
\text { antibiotics and so on, and non-attendance at appointments } \\
\text { in hospital clinics and primary care). }\end{array}$
\end{tabular}

Evidence of mental illness which is either current, recurrent or previous but severe.

Most commonly any such mental illness will be depression, which can vary widely in severity. The following count as severe, even if currently stable:

- bipolar affective disorder

- $\quad$ schizophrenia or other psychotic illness

- learning disability or other cognitive impairments

Previous self-harm. Any episode within the last five years or a life total of more than five episodes should act as factors to trigger psychiatric referral.

For live donors:

Evidence of any of the above factors affecting potential donors as well as recipients; or

Evidence of significantly dysfunctional family relationships, particularly between the recipient and prospective donors.

\section{Identifying the need for additional intervention}

Psychiatric assessment of donors and recipients is not simply a matter of selection: it might identify a need for additional intervention before or alongside surgery, or for additional support after it. Donors whose risk of depressive relapse is raised, but not so much as to preclude donation, may clearly need closer monitoring of mood after the transplant, possibly coupled with the pre-emptive (or at least early) use of antidepressants. Similarly, the assessment may identify a mental disorder in a recipient which might preclude current listing, but which is amenable to treatment, and therefore does need not prevent listing in the future. Examples include a significant needle phobia or major (but temporary) noncompliance arising from an untreated depressive illness.

\section{MANAGEMENT}

Before a transplant the psychiatrist's role requires highly specialised joint work with transplant teams, and close contact with general adult psychiatry and addiction services. After the transplant the role is closer to that of a liaison psychiatrist, especially in the early period.

\section{Peri-transplantation}

Any transplant is a major surgical procedure, and by definition undertaken on an already unwell patient. The risk of delirium is therefore high, and transplant recipients also run the risk of the well-known psychiatric effects of corticosteroids as well as the less familiar neurotoxic and psychiatric side effects of the immunosuppressants cyclosporin and tacrolimus. The principles of diagnosis and management are no different in the transplant setting than any other intensive care unit, high dependency unit or post-operative ward.

\section{Post-transplantation}

Major surgery and intensive therapy unit (ITU) stays confer a risk of post-traumatic stress disorder and adjustment disorder. This is no less true after transplantation, particularly when complications ensue. Transplant recipients also have to deal with the knowledge that their bodies contain an organ that previously belonged to a now-dead stranger or a still-living relative, friend, spouse or altruistic donor. Not surprisingly, some find this difficult. Beliefs about the donor are sometimes incorporated into the delusions and hallucinations of delirium, and it is not uncommon to encounter recipients who attribute changes in their outlook after transplant to being 'taken over' by the personality of the donor.

Transplant recipients also differ from other ITU survivors in their requirement for life-long immunosuppression and medical monitoring, as well as the ever-present threat of rejection, graft failure and consequent return to dialysis or the prospect of retransplantation. Anxiety and mood disorders are therefore common. These disorders require attention in their own right, especially when they are associated with poor adherence to treatment or a relapse of the alcohol or drug misuse that led to the original need for transplantation. Transplant psychiatrists therefore need to be available to the clinicians who run post-operative transplant clinics.

\section{CONCLUSION}

Transplant units have developed reliance upon the small and highly specialised field of transplant psychiatry to assist them in maximising transplant outcomes. The psychiatrists undertaking this work need a familiarity with transplant procedures and the relevant legislation, as well as a thorough grounding in the practice of psychiatry both in the general hospital and the wider community. Practising this work successfully is necessarily interdisciplinary. 


\section{KEY POINTS}

- The need for transplantation is often generated, directly or indirectly, by mental disorder. This is especially true in the case of liver disease.

- Problematic non-compliance and behavioural risk factors are not in themselves mental disorders, but may indicate a need for psychiatric or psychological assessment as part of a transplant work-up.

- The aim of pre-transplant assessment of recipients is two-fold: to select for those with the best potential outcomes and to identify any need for additional support or intervention before or after surgery.

- Pre-transplant psychiatric assessment of potential live donors has the same dual aim; in the case of altruistic donors it is required by law.

- Psychiatric assessment in this area is increasingly, and necessarily, protocol-guided, although protocols must be interpreted using sound clinical judgement about their applicability to individual cases.

\section{FURTHER READING}

- Anantharaju A, van Thiel DH. Liver transplantation for alcoholic liver disease. Alcohol Res Health 2003; 27:257-68.

- Bathgate AJ, UK Liver Transplant Units. Recommendations for alcohol-related liver disease. Lancet 2006;367:2045-6.doi:I0.I0I6/ S0I40-6736(06)68904-6

- DiMartini A, Crone C, Fireman M et al. Psychiatric aspects of organ transplantation in critical care. Crit Care Clin 2008; 24:949-8I. doi:10.1016/j.ccc.2008.05.001

- $\quad$ Potts SG. Assessing altruistic donation. Br J Renal Med 2009;14:26-8.

- Trzepacz PT, DiMartini A, editors. The transplant patient: biological, psychiatric and ethical issues in organ transplantation. Cambridge: Cambridge University Press; 2000.

- UK Liver Transplant Group. Recommendations for liver transplant assessment in the context of illicit drug use. Bristol: UK Transplant; 2007. Available from: http://www.uktransplant.org.uk/ukt/about_ transplants/organ_allocation/pdf/uk_liver_transplant_group_ recommendations_for_liver_transplant_assessment_illict_drug_ use-2007.pdf

\section{SELF-ASSESSMENT QUESTIONS}

I. A 58-year-old former nurse enquires about altruistic donation of one of her kidneys. Which one of the following pieces of advice is most appropriate?

A. She is too old for such a procedure.

B. Psychiatric assessment is advisable as the last step in preparation for the transplant.

C. Psychiatric assessment is advisable before any invasive investigations.

D. Psychiatric assessment is required by law before any invasive investigations.

E. Outcome for the recipient is comparable to that of cadaveric donation.

2. A 25-year-old man presents with fulminant hepatic failure after his second paracetamol overdose, taken when intoxicated with alcohol during a row with his girlfriend. Which one of the following is true?

A. Urgent liver transplant is absolutely contraindicated on the grounds of recurrent self-harm.

B. Urgent liver transplant is absolutely contraindicated on the grounds of current substance misuse.

C. Urgent liver transplant is absolutely contraindicated if he declines it, and assessment identifies no mental disorder.

D. There are relative contraindications (recurrent selfharm, alcohol misuse) and together these amount to an absolute contraindication on the basis of this information alone.

E. Cadaveric liver transplantation is absolutely contraindicated, but an offer of live liver lobe donation from his girlfriend might be acceptable.
3. Psychiatric assessment is widely undertaken in potential candidates for liver transplant to treat alcoholic liver disease (ALD). Which one of the following statements is most correct?

A. Psychiatric assessment adds little because rates of relapse into alcohol misuse are very high.

B. A six-month period of abstinence from alcohol is mandatory: without it transplantation cannot take place.

C. A six-month period of abstinence from alcohol is sufficient: no further psychiatric assessment is necessary.

D. Outcomes are worse in transplantation for ALD than for other forms of liver disease, and so patients with ALD should have a lower priority on transplant lists.

E. Graft and patient survival after transplant for ALD is good if patients are carefully selected.

4. A diabetic patient has developed end-stage renal disease owing to diabetic nephropathy. He has been poorly compliant with the management of his diabetes, and his glucose has run too high for years. He requests a referral for a kidney transplant. Which one of the following is applicable?

A. Non-compliance is a form of mental disorder and requires psychiatric referral.

B. Non-compliance may in itself be a contraindication to transplant listing, with or without psychiatric assessment.

C. Non-compliance is irrelevant if the patient's sister comes forward wishing to donate her kidney.

D. Non-compliance cannot be focal: non-compliance in one area predicts non-compliance in others.

E. Psychological treatment for non-compliance is ineffective unless there is mental disorder. 
5. A 53-year-old man requires haemodialysis for end-stage renal disease caused by the lithium he has been prescribed for more than $\mathbf{2 0}$ years for bipolar disorder. Which one of the following statements is most applicable?

A. Bipolar disorder of this severity and duration is incompatible with successful transplantation.

B. The lithium must be stopped.

C. Post-operative management of the patient's mood lies with the transplant psychiatrist.
D. Lithium is a rare cause of end-stage renal disease.

E. Changing to an alternative mood stabiliser carries substantial risks and should be avoided.

This paper was originally published as part of the Psychiatry module in the RCPE Online Continuing Medical Education Programme. Online CME, including the anwers to these questions and further material, is available to Fellows and Members at: https://members.rcpe.ac.uk/cme.php

\section{DATES FOR YOUR DIARY: FORTHCOMING SYMPOSIA IN 2010}

RCPE Northern Ireland Symposium:

Updates in Medicine

(Hilton Hotel,Templepatrick)

28 January

- Hot Topic Symposium:

Healthcare-associated Infection

2 February

- RCPE Dundee Symposium:

Moving Points in Medicine

(Ninewells Hospital and Medical School)

10 February

- Respiratory Medicine Symposium 25 February

- RCPE Aberdeen Symposium:

'Medical Interfaces' Series, Year 5

(Medical School, University of Aberdeen) 10 March

\begin{tabular}{lr}
\hline Oncology Symposium & 6 May \\
\hline Infectious Diseases Symposium & 11 June
\end{tabular}

- RCPE Yorkshire Symposium

(Bradford Royal Infirmary)

25 June

a Care of the Elderly Symposium 15 September

- Paediatric Symposium

(RCPE/RCPCH joint symposium)

23 September

- Diabetes and Endocrinology Symposium 7 October

- Collegiate Members' Symposium 22 October

- Neurology Symposium

27 October

Renal Medicine Symposium

(SRA/RCPE joint event)

5 \& 6 November

\begin{tabular}{ll}
\hline Gastroenterology Symposium & 11 November \\
\hline Cardiology Symposium & 23 November
\end{tabular}

- 50th St Andrew's Day Festival Symposium:

Updates in Acute Medicine 2 \& 3 December
All symposia are held at the Royal College of Physicians of Edinburgh unless otherwise stated.

Programme details will become available at: http://events.rcpe.ac.uk

Contact the Education Assistant (tel:013I 225 7324, email: h.elliott@rcpe.ac.uk) to be added to the mailing list for an event.

Unable to attend a particular symposium? Selected lectures (more than 100 currently available) can be listened to in the Online Education section of the secure area of the College website. Log on at http://www.rcpe.ac.uk. If you have lost or forgotten your username or password please contact webmaster@rcpe.ac.uk.

Online booking is now available at http://events.rcpe.ac.uk 